\title{
Finding Object Categories in Cluttered Images Using Minimal Shape Prototypes
}

\author{
Johan Thuresson and Stefan Carlsson \\ Numerical Analysis and Computing Science, Royal Institute of Technology, (KTH), \\ S-100 44 Stockholm, Sweden
}

\begin{abstract}
We present an algorithm for recognizing object categories as opposed to specific instances, based on matching prototypical object shapes to gray-level images. The central part of the algorithm is the establishment of correspondence between prototype template and image based on finding qualitative shape invariants in the form of order types of sets of points and lines. A central problem of any matching algorithm like this is the rejection of background and foreground clutter in the image resulting in erroneous matches. By deforming the prototype and iterating the computation of correspondence we reject outliers and improve the quality of the matching. Experimental results in terms of locating examples of specific object classes in real gray-level images are presented. The results demonstrate the robustness of the algorithm and make it an interesting candidate for any categorical recognition system such as database indexing.
\end{abstract}

Keywords: Object recognition, shape, correspondence

\section{Introduction}

Recognizing categories or classes of objects in an image such as cars, humans, horses etc. as opposed to specific instances is a complex problem due to their variation of appearance in the image. This variation has multiple sources. By design, an object category can vary in shape, colour, texture etc. Any algorithm that tries to find an object category therefore has to deal with this variation. This can be done by identifying what is common to all instances and neglect the variation. It is generally considered that object shape is the property that is most preserved among instances. With this we mean that variation of shape is relatively smaller than the variation of colour e.g. This rule of course has exceptions, such as fruit categories where colour can be a strong invariant.

In this paper we will use the shape projected by a $3 \mathrm{D}$ object in an image as a cue for recognition. This means that we have to deal specifically with variation of projected object shape. This variation has two main sources:

- variation of the $3 \mathrm{D}$ shape within the category

- variation of object 3D location relative to the camera 
The projected shape variation of a certain category can therefore be substantial making the recognition problem very complex. This means that we will need an excessive number of shape templates if we want to find a match. In order to reduce this complexity we will use a shape matching algorithm that is tolerant to the projected variation to a large degree. In this paper we will concentrate on the variation due to the variation of 3D shape within the category. The essential problem of matching shapes by finding correspondences, is that of finding invariants, i.e. properties that remain the same in spite of the shape variation.

We will refer to the algorithm for finding correspondences as the shape matching and it plays a central role in the recognition problem. The procedure which is basically the idea of geometric hashing [7 can be shown to be equivalent to the "Matching with Shape Contexts" as presented in [1] and applied to the problem of character recognition. The idea of recognition using deformable templates goes back a long way [2], [3] but has never been applied to the problem of categorical recognition in cluttered gray-level images. Most work in this area assumes that clutter has already been removed [5, 6], 8], [10].

\section{Shape Matching}

For the purpose of finding correspondences we will use the algorithm sketched in [4] and used for human pose estimation in [9]. This algorithm produces point to point correspondence between sets of oriented elements, i.e. sets of locations (points) with orientations (lines). These sets can be obtained by performing Canny-Deriche edge detection, edge linking, and equidistant subsampling of location and orientation. Figure 1 depicts two such instances of rabbits.

Consider complexes of four oriented elements from two shapes when these are in "perceptual correspondence", $a_{1}-b_{1}, a_{2}-b_{2}, a_{3}-b_{3}, a_{4}-b_{4}$. Then the order of locations as they are traversed e.g. clockwise is preserved. The order of orientation is also preserved. And for each line and two points, the way the line intersects relative to the two points (between or not) is preserved.
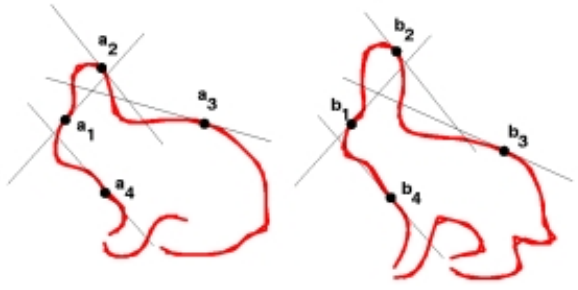

Fig. 1. Point line complexes with same topological type

Each unique combination of these three order types is given a unique index. Correspondence between two shapes can then be computed by the procedure: 
- compute oriented elements of two shapes $A$ and $B$.

- for each combination of four points $a_{1}, a_{2}, a_{3}, a_{4}$ in shape $\mathrm{A}$ and $b_{1}, b_{2}, b_{3}, b_{4}$ in shape $B$, compute the order type index

- whenever two sets $a_{1}, a_{2}, a_{3}, a_{4}$ in shape $\mathrm{A}$ and $b_{1}, b_{2}, b_{3}, b_{4}$ in shape $B$ have the same order type index, vote for the correspondence between $a_{1}-b_{1}, a_{2}-$ $b_{2}, a_{3}-b_{3}, a_{4}-b_{4}$ and update a voting matrix

- after all combinations of four points in the two shapes have been traversed, find a unique assignment between points in shape $A$ and shape $B$ using the greedy algorithm on the accumulated voting matrix

The voting matrix mentioned previously contains information about the relations between prototype and image elements. Each entry states how strong a specific prototype element corresponds to a specific image element.

\section{Recognition in Clutter}

The shape matching algorithm presented can be seen to work well as long as the two shapes are clean with no major background or foreground clutter. 4], [9]. Introducing clutter into the image will result in erroneous correspondences that have to be corrected. We will do this in an iterative way as depicted by the flowchart of figure 2

Recognition is achieved by matching a shape prototype of a certain object category to gray-level images containing instances of that category. The shape prototype is deformed using correspondences found between the shape prototype and the image. After deformation, the image is validated against the deformed shape prototype to make a verdict if the object in the image belongs to the same class of objects as the shape prototype or not.

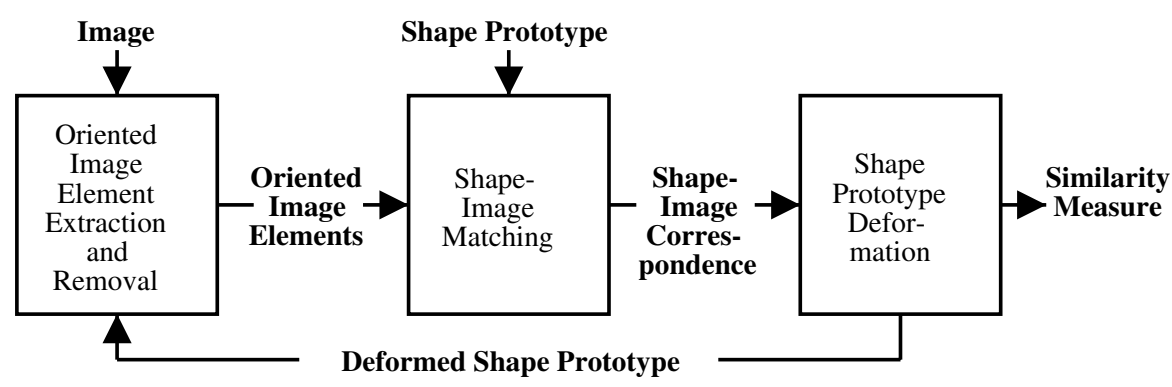

Fig. 2. Flowchart of the algorithm

Oriented Image Element Extraction and Removal: We start with an image $B$ and a set of oriented elements $a_{i}$ that belong to a shape prototype $A$. A 
number of oriented elements $b_{j}$ are then extracted in the image $B$. Each iteration correspondences between shape prototype and image are computed and used to estimate a deformation $T$ of a certain parametric type. After the first iteration of the algorithm, the shape prototype has been deformed according to $A^{\prime}=T(A)$. If the correspondences are correct, this deformation has brought the prototype close to the image of the object and we can tell where in the image the object we are looking for is positioned. The oriented image elements that do not belong to the object can thus be rejected.

This rejection is performed as follows. First oriented elements $\hat{b}_{j}$ are extracted in the image $B$. Then the oriented image elements whose distances to the closest element of the deformed shape prototype $A^{\prime}$ exceed a threshold $t, d\left(\hat{b}_{j}, A^{\prime}\right)>t$, are deleted. $t$ is constant and manually chosen to give a reasonable amount of oriented elements for all images.

Elements are also deleted due to angle difference. If none of the oriented shape prototype elements inside the range $t$ differs from an image element with less than the angle $u$, it is deleted. After both rejections we have a new set of oriented image elements $b_{j}$.

The edge removal distance threshold $t$ is lowered each iteration, resulting in a more and more 'clean' image. The angle threshold $u$ is however constant.

For each iteration we want to use the maximum number of allowed image points. As points in areas distant from the prototype are removed, points in its proximity can be sampled more densely. Therefore the points have to be resampled each iteration.

In figure 3 we see the oriented image elements operated on. And in figure 4 we see the iteration steps of the edge removal and correspondence refinement.

Shape-Image Matching: Using the oriented image elements remaining after the removal process, a set of correspondences $\left(a_{i_{r}}, b_{j_{r}}\right) \in C$ are computed by the shape matching algorithm described previously. This produces $1-1$ correspondences between prototype and image elements.

Shape Prototype Deformation: The correspondences that now have been computed are used to deform the shape prototype. In the first iteration of the algorithm the shape prototype is deformed using isotropic scaling. In the second iteration the shape prototype is deformed using anisotropic scaling. In the third iteration, different parts of the shape prototype are deformed individually, using anisotropic scaling. A part is a subset of the shape prototype elements.

The deformation of each iteration is carried out in the following way, using the least square method. Deform the model using the correspondence set $C$; delete the correspondence $\max _{r} d\left(a_{i_{r}}^{\prime}, b_{j_{r}}\right)$ from the correspondence set $C$, i.e. the correspondence whose oriented elements are most distant from each other. For the isotropic scaling, this is repeated until only a few correspondences remain. However, for the anisotropic scaling, this is repeated until the maximum correspondence distance falls below a specified threshold. This threshold is used, because anisotropic scaling with few correspondences can cause the prototype to drift off of target. The threshold is chosen manually after observation of examples. 


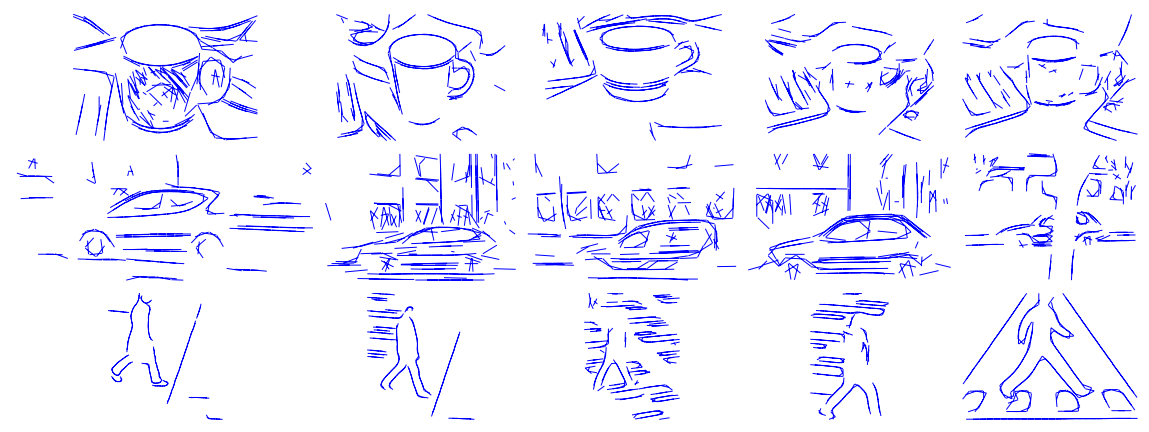

Fig. 3. Oriented Image Elements
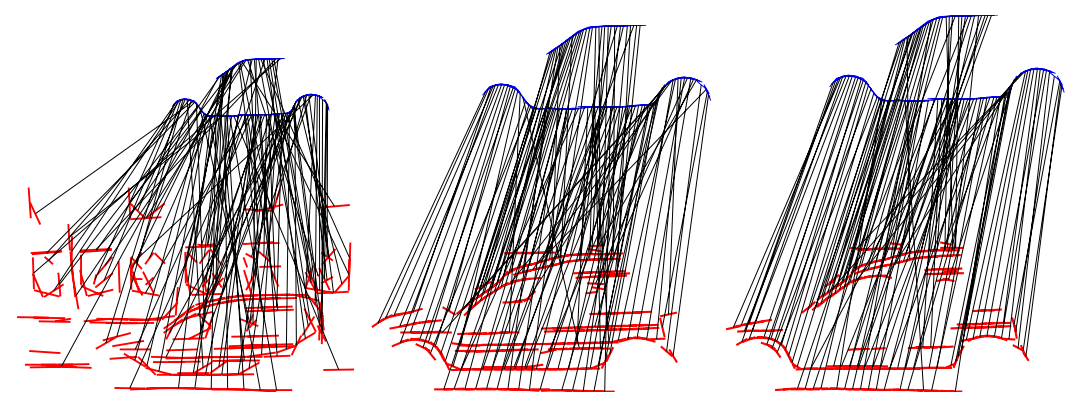

Fig. 4. Iteration steps of element removal and correspondence refinement

Table 1. Similarity Measures

\begin{tabular}{l|lll}
\hline & $\begin{array}{l}\text { Mug } \\
\text { Model }\end{array}$ & $\begin{array}{l}\text { Car } \\
\text { Model }\end{array}$ & $\begin{array}{l}\text { Walker } \\
\text { Model }\end{array}$ \\
\hline Mug1 & $\mathbf{. 9 2}$ & .46 & .41 \\
Mug2 & $\mathbf{. 9 4}$ & .00 & .29 \\
Mug3 & $\mathbf{. 8 5}$ & .00 & .12 \\
Mug4 & $\mathbf{. 7 1}$ & .00 & .00 \\
Mug5 & $\mathbf{. 8 3}$ & .00 & .35 \\
\hline Car1 & .00 & $\mathbf{. 7 2}$ & .20 \\
Car2 & .00 & $\mathbf{. 8 5}$ & .35 \\
Car3 & .00 & $\mathbf{. 7 6}$ & .28 \\
Car4 & .31 & $\mathbf{. 9 0}$ & .27 \\
Car5 & .30 & $\mathbf{. 6 0}$ & .40 \\
\hline Walker1 & .09 & .27 & $\mathbf{. 5 7}$ \\
Walker2 & .08 & .00 & $\mathbf{. 5 8}$ \\
Walker3 & .33 & .00 & $\mathbf{. 5 7}$ \\
Walker4 & .33 & .00 & $\mathbf{. 5 9}$ \\
Walker5 & .18 & .00 & $\mathbf{. 6 8}$ \\
\hline
\end{tabular}



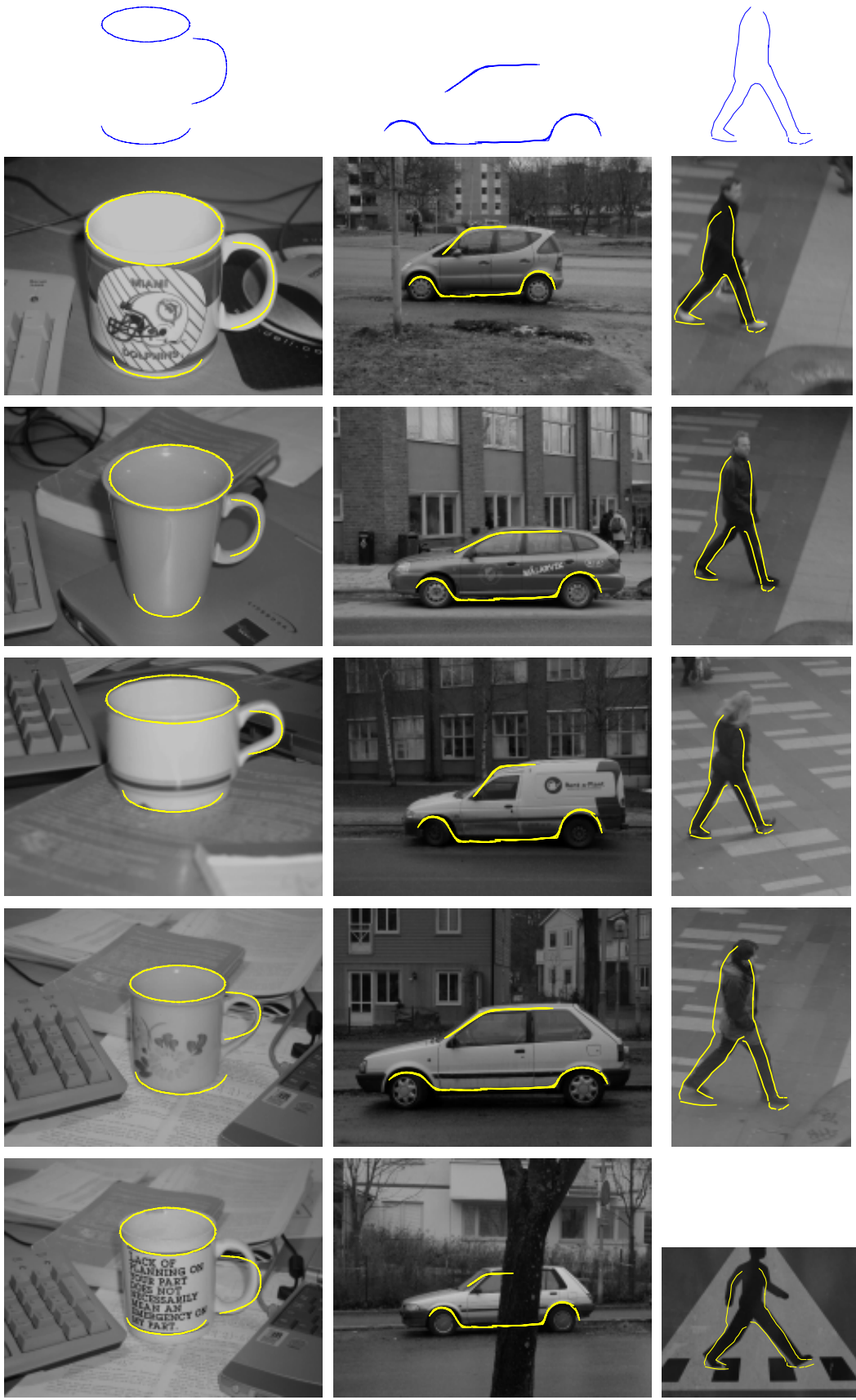

Fig. 5. Shape prototypes, original and deformed 
This method is not invariant to rotation in the image plane. To make the it invariant, each prototype could be matched at different angles.

Similarity Measure Computation: After three iterations of the algorithm, a similarity measure is computed. The measure is the number of oriented shape prototype elements that are in the proximity of at least one oriented image element, divided by the total number of oriented prototype elements. In the cases where the deformation becomes unrealistic, the similarity measure is however set to zero.

\subsection{Shape Prototype}

The deformation of the shape prototype works because of its simplicity. A more complex shape prototype would have needed a more complex deformation to succeed in fitting all parts of the shape prototype to their corresponding parts of the object in the image. However this increases the risk of getting false positives, because if the prototype is easy to deform, it can more easily be deformed to parts of the image that do not belong to the object to be found. The shape prototype should in addition to being simple, be general, i.e. it should not contain parts present only in a few instances of the object class represented. It should also be typical, or have an 'average' shape for the class represented, meaning that as little deformation as possible should be needed to fit the shape prototype to all shapes of the class. Furthermore for the deformation to be possible to perform on ordinary gray-level images, the shape prototype should not contain any elements that are typically hard to extract from images.

Even though the principle is valid that the simpler the model, the easier the match, we do not want the model to be too simple. If so, the risk of having false matches increases as well, because you can find shape prototype present in an image, even though the object is not.

Summing up the shape prototype should only contain the lowest common denominator of edges that are easily extractable in an image without being mistaken for something else.

\section{Results}

We have tested the algorithm on three different categories: mugs, cars and walkers. The results are satisfactory as can be seen in figure 5. We have succeeded to deform the shape prototypes to the image objects of their respective classes.

The shape prototypes are extracted from good representative images, and can be seen at the top of figure 5. The mug shape prototype, is divided into three parts: top ellipse, bottom half-ellipse, and ear. The car shape prototype is divided into a top and bottom part. And the walker shape prototype is divided into a left and right part.

In table 1 the similarity values are presented for all combinations of shape prototypes and images. In the table we can see that the correct matches, which are in bold face type, are dominant both horizontally and vertically in the table. 
All true matches have a similarity measure of more than .50 and all false matches have a similarity measure of less than .50. Thus we can use this value as a threshold to judge the class of an object.

The complexity of the algorithm is $O\left(n^{4}\right)$, and in order to limit time, we have chosen to use maximum 200 per set of oriented elements.

\section{Conclusion}

There are some cases of instability of the algorithm. Firstly it is sensitive to bad lighting conditions. This is due to complexity reasons. If the edge detection threshold is lowered, the coarseness of the oriented image elements is increased, not to exceed the maximum number of allowed elements. A coarse set of oriented image elements makes it hard to perform recognition however.

Secondly the algorithm is sensitive to the object taking up to small a part of the image. In that case a small number of the oriented image elements will belong to the car, and it will have low resolution.

A third problem is the dependence of the algorithm on viewing angle. The cars and mugs are seen from basically the same angle. A future enhancement to cope with this problem would be to use epipolar geometry.

However the strength of the algorithm is that it works on ordinary images. It is not dependent on a segmentation algorithm that cuts out a silhouette of the object for us.

\section{References}

1. Belongie S. and Malik J. Matching with Shape Contexts Proc. 8:th International Conference on Computer Vision (ICCV 2001)

2. Bremermann H. J. Cybernetic Functionals and Fuzzy Sets. IEEE Systems, Man and Cybernetics Group Annual Symposium, pages 248-254, 1971.

3. Burr D. J., Elastic Matching of Line Drawings, IEEE Trans. on Pattern Analysis and Machine Intelligence, 3, No. 6, pp. 708-713, November (1981)

4. Carlsson. S, "Order Structure, Correspondence and Shape Based Categories", International Workshop on Shape Contour and Grouping, Torre Artale, Sicily, May 26-30 1998, Springer LNCS 1681 (1999)

5. Cootes, T.F., Taylor, C.J., Cooper, D.H., Graham, J., Active Shape Models: Their Training and Application, CVIU(61), No. 1, January 1995, pp. 38-59.

6. Jain A. K, Fellow, IEEE, Zhong Y, and Lakshmanan S.: Object Matching Using Deformable Templates. IEEE Transaction on Pattern Analysis and Machinge Intelligence, Vol. 18, No. 3, March

7. Lamdan Y., Schwartz, and . Wolfson, Object recognition by affine invariant matching. In: Proc. CVPR-88, pp. 335-344. (1988)

8. Sclaroff S., Deformable Prototypes for Encoding Shape Categories in Image Databases, PR(30), pp. 627-641. No. 4, April (1997),

9. Recognizing and Tracking Human Action J. Sullivan and S. Carlsson, Proc 7:th European Conference on Computer Vision (ECCV), Copenhagen, Denmark 2002. (PDF )

10. Yuille, A.L., Deformable Templates for Face Recognition, CogNeuro(3), No. 1, 1991, pp. 59-70. 